\title{
Robot-assisted laparoscopic partial nephrectomy is a safe and effective option for clinical T2 renal cell carcinoma: a case-series from single-institution
}

\author{
Gongwei Long ${ }^{1,2 \#}$, Man Liu ${ }^{1,2 \#}$, Yucong Zhang ${ }^{1,2,3}$, Guoliang Sun ${ }^{1,2}$, Wei Ouyang ${ }^{1,2}$, Jun Yang ${ }^{1,2}$, \\ Zhihua Wang ${ }^{1,2}$, Zheng Liu ${ }^{1,2}$, Wei Guan ${ }^{1,2}$, Zhiquan $\mathrm{Hu}^{1,2}$, Shaogang Wang ${ }^{1,2}$, Heng Li $^{1,2}$ \\ ${ }^{1}$ Department of Urology, Tongji Hospital, Tongji Medical College, Huazhong University of Science and Technology, Wuhan, China; ${ }^{2}$ Hubei \\ Institute of Urology, Tongji Hospital, Tongji Medical College, Huazhong University of Science and Technology, Wuhan, China; ${ }^{3}$ Department of \\ Geriatrics, Tongji Hospital, Tongji Medical College, Huazhong University of Science and Technology, Wuhan, China \\ Contributions: (I) Conception and design: H Li, S Wang; (II) Administrative support: H Li; (III) Provision of study materials or patients: S Wang, \\ Z Hu; (IV) Collection and assembly of data: G Long, M Liu, Y Zhang, W Ouyang, G Sun; (V) Data analysis and interpretation: G Long, M Liu, J \\ Yang, Z Wang, Z Liu; (VI) Manuscript writing: All authors; (VII) Final approval of manuscript: All authors. \\ "These authors contributed equally to this work. \\ Correspondence to: Shaogang Wang; Heng Li. Department of Urology, Tongji Hospital, Tongji Medical College, Huazhong University of Science and \\ Technology, Wuhan 430030, China. Email: somethingnew@hust.edu.cn; lihengtjmu@163.com.
}

Background: Robot-assisted laparoscopic partial nephrectomy (RAPN) is increasingly used in the treatment of complex renal tumors, but it is still not commonly performed for T2 renal tumors and the reports of RAPN for renal tumors $\geq 7 \mathrm{~cm}$ were limited. Here we report our single-institution outcomes to evaluate the safety and efficacy of RAPN in treating T2 renal tumors.

Methods: This study was designed as a case-series study. We retrospectively reviewed our database and finally identified 16 patients undergoing RAPN for clinical T2 tumors. Seven patients who underwent standard laparoscopic partial nephrectomy (LPN) and 30 patients who were treated by radical nephrectomy $(\mathrm{RN})$ were included as controls. The baseline characteristics, perioperative outcomes, change of renal function, and oncological outcomes were analyzed.

Results: There was no significant difference between groups in baseline characteristics, intraoperative complications, and long-term oncological outcomes. Both RAPN and LPN had a longer operative time and higher blood loss compared with RN, but they could offer better renal function reservation. Two patients who underwent LPN converted to radical nephrectomy or open partial nephrectomy and no conversions happened in the RAPN group. The ischemia time seemed shorter in RAPN group but did not reach statistical significance (median 20.0 vs. $25.5 \mathrm{~min}, \mathrm{P}=0.118$ ). Except for blood transfusions, no other major complication was detected.

Conclusions: RAPN can provide fair preservation of renal function with acceptable major complications and oncological outcomes, therefore it is a safe and effective procedure for renal tumors $\geq 7 \mathrm{~cm}$. Its advantages over LPN still need to be ascertained by further studies with better design and larger sample sizes.

Keywords: Renal tumor; partial nephrectomy; T2; robotic surgical procedure

Submitted Jun 14, 2020. Accepted for publication Sep 18, 2020.

doi: $10.21037 /$ tcr-20-2324

View this article at: http://dx.doi.org/10.21037/tcr-20-2324 


\section{Introduction}

Renal cell carcinoma (RCC), which ranks second among the leading causes of deaths in patients with urologic tumors, is one the most frequently occurring cancers in Western communities $(1,2)$. Management of renal tumors has changed significantly in the last two decades, and partial nephrectomy $(\mathrm{PN})$ was recommended by guidelines for T1a renal tumors, whenever technically feasible $(3,4)$, as it allows preservation of renal function together with excellent cancer control $(5,6)$. For T1b renal tumors, PN still provides noninferior survival outcomes compared to radical nephrectomy $(\mathrm{RN})$ (7-13). Tumor size alone is no longer considered as a contraindication to $\mathrm{PN}$, and several centers have reported their PN experience for larger $(\geq 7 \mathrm{~cm})$ renal tumors (14-19). However, all these studies focused on open or laparoscopic PN (LPN).

Since the introduction of robotic da Vinci surgery, robot-assisted laparoscopic partial nephrectomy (RAPN) was being increasingly used, especially for more complex tumors, but the use of RAPN for T2 renal tumors was only reported by several studies from the USA and Europe (20-24). To provide relevant information about safety and oncological outcomes, especially originated from Asia, here we report our single-institution experience with RAPN for T2 renal tumors. We present the following article in accordance with the STROBE reporting checklist (available at http://dx.doi.org/10.21037/tcr-20-2324) (25).

\section{Methods}

\section{Ethical statement}

The study was conducted in accordance with the Declaration of Helsinki (as revised in 2013). The ethical approval of this study was exempted by the Ethics Committee of Tongji Hospital because of the retrospective and observational nature, and the data were de-identified. Informed consent was also not required due to the above reasons.

\section{Study design}

This study was designed as a retrospective study. All patients who underwent RAPN between February 2015 and November 2018 for localized renal tumors larger than $7 \mathrm{~cm}$ on preoperative imaging were included. The patients who underwent LPN for T2 tumors were also screened as control. Eventually, 16 patients who underwent RAPN and 7 patients who underwent LPN were identified in this study. Besides, as the $\mathrm{RN}$ is still the standard treatment for T2 RCC, another 30 patients with T2 RCC treated by RN were also included as a control.

Chest CT and blood biochemical tests were routinely conducted, and other examinations would also be conducted according to the symptoms for tumor staging. All of these patients had a preoperative abdominal computed tomography scan (CT) to assess the complexity of tumors. Magnetic resonance imaging (MRI) was performed when necessary for further evaluation. Based on the tumor complexity, patients were informed of the merits and demerits of different procedures and choose the procedure for themselves. All the RAPN were performed by the same surgeon who had an experience of more than 1,000 robotic surgeries.

\section{Data collection}

Baseline characteristics of patients, perioperative, pathological, and oncological outcomes were collected to evaluate the safety and efficacy of RAPN. The count data was described as the number of events, whereas the continuous variables were described as the median and interquartile range (IQR).

The collected baseline characteristics of patients included age, body mass index, gender, American Society of Anesthesiologists (ASA) score, size of renal tumor on CT (largest diameter in $\mathrm{cm}$ ), RENAL score (26), preoperative estimated glomerular filtration rate (eGFR), and preoperative CKD stage (27).

The assessed perioperative outcomes referred to operative time (OT), estimated blood loss (EBL), warm ischemia time (WIT), length of hospital stay, intraoperative and postoperative complications, blood transfusions, postoperative eGFR, overall mortality, cancer-specific mortality, local recurrence, and metastasis. The major complication was defined as Clavien grade $\geq 3$ (28).

The pathology outcomes encompassed histopathology, pathological tumor size, pT stage, Fuhrman grade, and margin status.

After surgery, the biochemical tests would be performed to assess the condition of electrolytes and renal function. Patients would be advised to take examinations every 3 to 6 months for the first 2 years after surgery, including physical examinations, biochemical tests, ultrasound, CT scan, or further investigations according to the practical circumstances. 
Table 1 Characteristics of patients and tumors

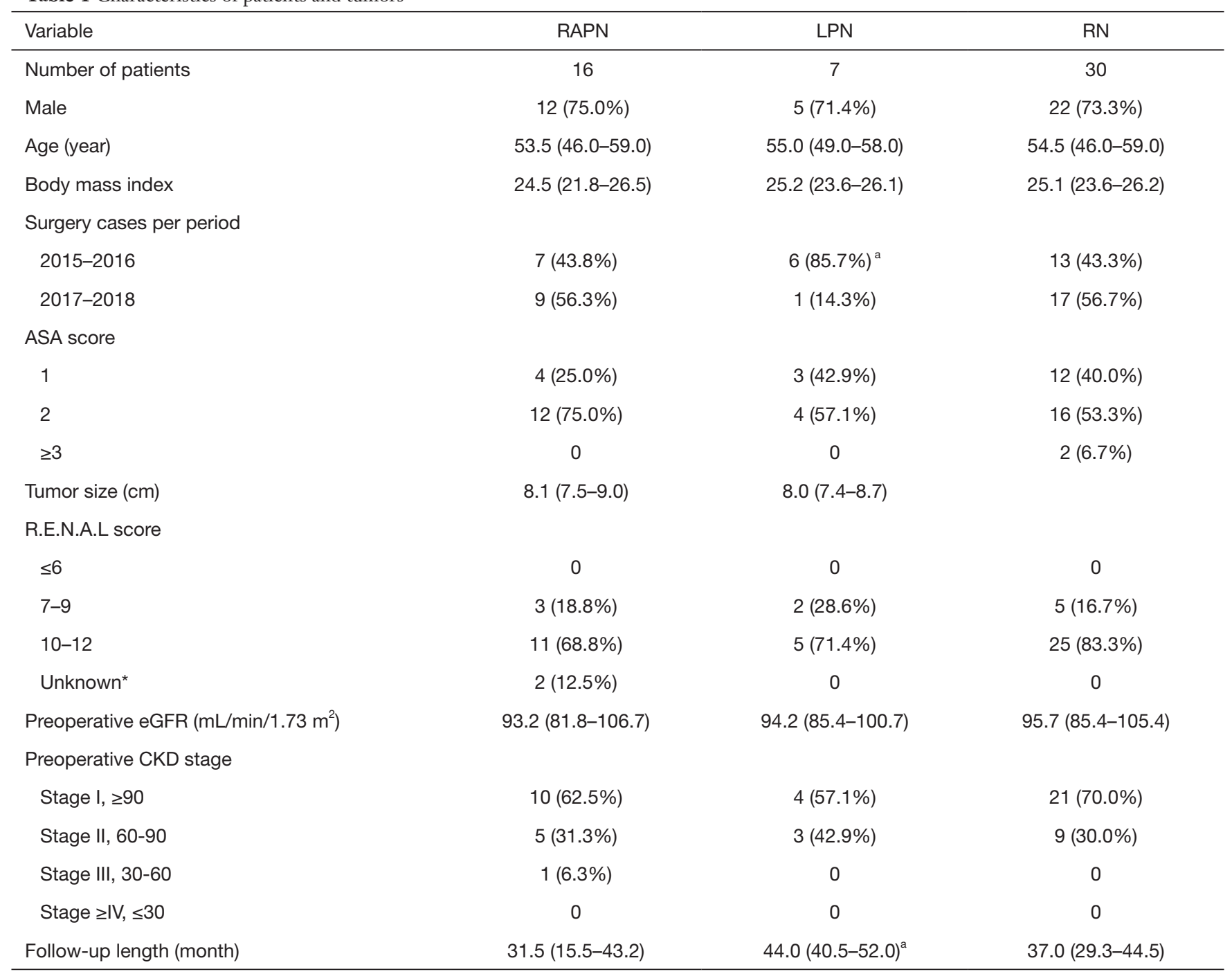

Continuous data was presented as median (interquartile range) and count data was presented as $\mathrm{n}(\%) .{ }^{*}$, the original radiologic data was unavailable. ${ }^{a}$, significantly different from other two groups. RAPN, robot-assisted laparoscopic partial nephrectomy; LPN, laparoscopic partial nephrectomy; RN, radical nephrectomy; ASA score, American Society of Anesthesiologists score; CT, computed tomography; eGFR, estimated glomerular filtration rate; CKD, chronic kidney disease.

\section{Statistical analysis}

SPSS 25.0 was used to conduct all the statistical analysis in this study. The relevant outcomes of different groups were compared, and the Student's t test was used for continuous data while Fisher's exact test was applied for count data. P value $<0.05$ was considered statistically significant.

\section{Results}

Eventually, 16 RAPN patients, 7 LPN patients, and 30
RN patients were included in this study. The baseline characteristics of the patients were listed in Table 1 . The baseline age, proportion of gender, ASA score, and preoperative renal function were similar among these groups. The CT scan and MRI were retrieved for the assessment of the size and complexity of tumors (Figure 1). The tumor size and tumor complexity were also similar for the RAPN group and LPN group. The RN groups had the most patients with a RENAL score $>9$, but the difference was not statistically significant. Notably, two patients 

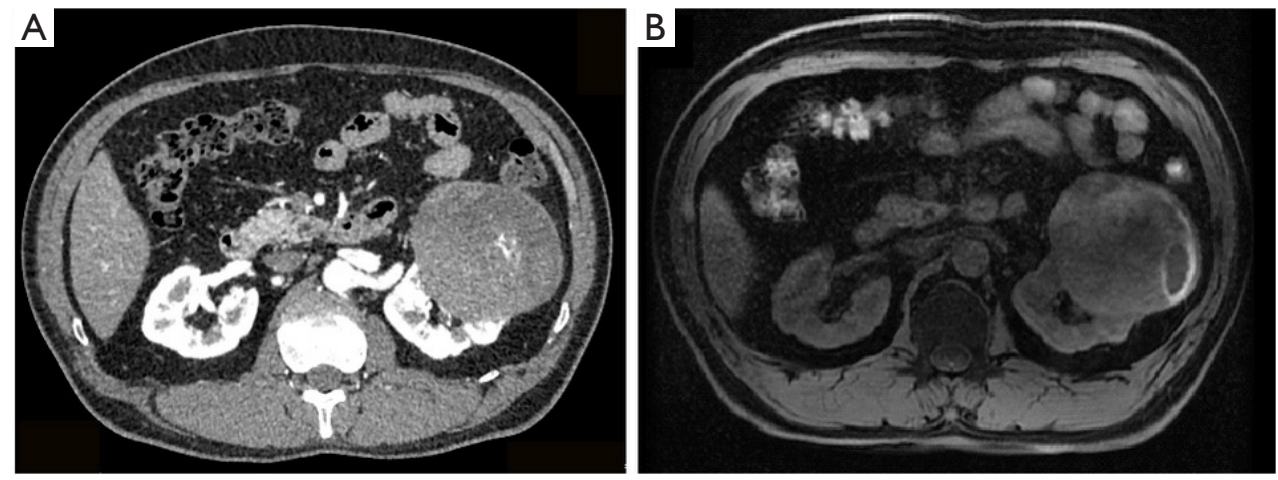

Figure 1 The representative radiological imaging of a patient with a lesion on the left kidney. (A) The CT scan of the kidney; (B) the MRI scan of the kidney.

in the RAPN group had a CT scan in a local medical institution and the tumor sizes were documented in the CT report, but the original imaging data were unavailable. Therefore, the assessment of tumor complexity was unable to be conducted. Most LPNs (6 of 7) for T2 tumors were conducted during 2015 and 2016 while RAPNs were mostly conducted during 2017 and 2018.

All RAPNs were performed successfully without conversion to RN or open surgery while one LPN was converted to $\mathrm{RN}$ and one was converted to open PN. The relevant perioperative outcomes were demonstrated in Table 2. The OT and EBL were significantly lower in the $\mathrm{RN}$ group than RAPN group (OT: $\mathrm{P}<0.001, \mathrm{EBL}$ : $\mathrm{P}<0.001)$ and LPN group (OT: $\mathrm{P}<0.01, \mathrm{EBL}$ : $\mathrm{P}<0.001$ ), but the OT and EBL did not differ between the RAPN and LPN groups. The LPN seemed related to a longer warm ischemia time [25.5 (IQR, 21.5-29.5] min vs. 20.0 (IQR, 18.0-25.0) min], though not statistically significant $(\mathrm{P}=0.118)$. Blood transfusions were performed for 2 patients during the surgery for both RAPN and LPN groups and 3 patients in the $\mathrm{RN}$ group, and no other major perioperative complications happened.

After assessing the pathological reports, 15 RAPN patients and all LPN and RN patients were identified as pT2. One patient in the RAPN group with two lesions on the right kidney was identified as pT1b, as the $7.8 \mathrm{~cm}$ lesion on the lower right kidney was pathologically confirmed as a benign cyst. Pathological classification and Fuhrman grade were examined, and no significant difference was found among these groups. Most tumors were confirmed as clear cell carcinoma or papillary carcinoma and most were graded as Fuhrman grade II. One tumor in the RAPN group was classified as Fuhrman grade III clear cell carcinoma. The other 1 patient was diagnosed as high-grade RCC and hard to be pathologically classified. No positive surgical margin was reported for all patients and the median hospital stay was 8.0 (IQR, 7.8-9.0) days for RAPN, 8.0 (IQR, 7.5-9.0) days for LPN, and 8.0 (IQR, 7.3-9.0) days for LPN.

The eGFR at 1 day and 3 months after surgery were examined to assess the postoperative renal function. The median postoperative eGFR at 1 day and 3 months after surgery were similar for these groups. The decreased eGFR and CKD upstaging were also comparable among these groups. The decreased eGFR was significantly higher in the $\mathrm{RN}$ group than the RAPN group $(\mathrm{P}=0.024)$ and $\mathrm{LPN}$ group $(\mathrm{P}=0.038)$, but the $\mathrm{CKD}$ upstaging was comparable among the three groups.

The median follow-up was 31.0 (IQR, 15.5-30) months for the RAPN group, 44.0 (IQR, 40.5-52.0) months for the LPN group, and 37.0 (IQR, 29.3-44.5) months for the RN group. The follow-up length was longer in the LPN group compared with the RAPN group $(\mathrm{P}=0.011)$ and the $\mathrm{RN}$ group $(\mathrm{P}=0.014)$. No patient died during the follow-up and no local recurrence was detected. The patient pathologically diagnosed as high-grade RCC in the RAPN group had enlarged perihepatic lymph nodes in the preoperative abdominal CT examinations. He was treated with sunitinib after surgery and peritoneal metastases were detected by PET-CT 13 months later.

\section{Discussion}

$\mathrm{PN}$ is currently the gold standard of surgery for feasible T1 renal tumors, and it has been proven as effective as RN on cancer control $(5,6)$. And retrospective studies have suggested that even for $\mathrm{T} 2$ tumors, its efficacy would not 
Table 2 Perioperative and oncological outcomes

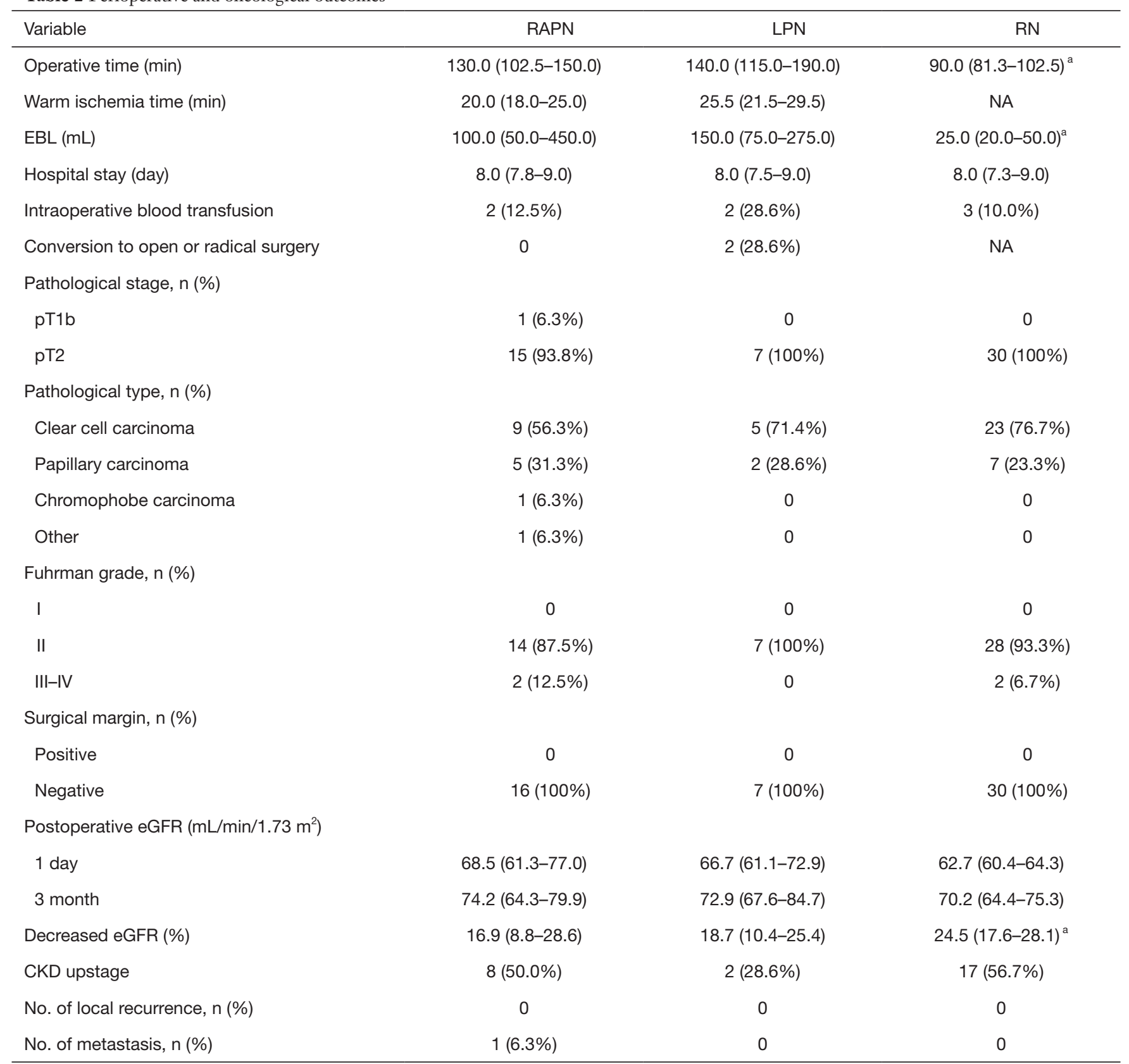

Continuous data was presented as median(interquartile range) and count data was presented as $\mathrm{n}$ (\%). ${ }^{\text {a }}$, significantly different from other two groups. RAPN, robot-assisted laparoscopic partial nephrectomy; LPN, laparoscopic partial nephrectomy; RN, radical nephrectomy; EBL, estimated blood loss; eGFR, estimated glomerular filtration rate; CKD, chronic kidney disease; NA, not applicable.

be compromised (29,30). PN for clinical T2 renal tumors was reported by several studies but most of these studies were limited to open surgery or standard laparoscopic approach (30). As for RAPN, only a few studies from the U.S.A and Europe showed the relevant outcomes (20-24). Herein, we report our single-institutional experience with RAPN for renal tumors $\geq 7 \mathrm{~cm}$. To the best of our knowledge, this is the first study that originated from Asia that represents the outcomes of RAPN for T2 renal tumors.

Consideration of the complications is essential when high-risk surgical procedures are performed. The only intraoperative complication of RAPN in our study was a 
blood transfusion and the transfusion rate was similar to other reports of RAPN for T2 renal tumors. As for the postoperative complications, bleeding and urinary leak were mostly reported (20). But none of these complications happened among our patients and it might because of the use of the two layers, continuous, unknotted renorrhaphy technique (31).

Reducing renal function loss is the main advantage of PN. Compared with radical surgery, PN can provide better preservation of renal function and therefore might be more beneficial for the overall survival and cancer-specific survival $(32,33)$. In our study, no patient received dialysis after surgery. Three months after surgery, 8 of 16 RAPN patients experienced an upward migration of CKD stage and only one patient migrated to CKD stage III with a median eGFR change of 14.9 (IQR, 8.7-24.1) $\mathrm{mL} / \mathrm{min} / 1.73 \mathrm{~m}^{2}$. The proportion of CKD migration was higher than Malkoc et al.'s study (22) (10 of 54 patients). One possible reason is that our long-term renal functions are based on the 3-month data after surgery. Compared to the report of Bertolo et al. (20), the eGFR loss at the discharge of our report was also higher (27.0\% vs. $15.6 \%)$. The larger tumor sizes and higher tumor complexity of our study might be the sources of difference. Besides, the resection strategy is closely associated with the postoperative renal function (34). In the present study, the tumors were resected about $5 \mathrm{~mm}$ from the edge of the tumor. When the tumors are next to the collecting system, to avoid the possible injury, the resection thickness might be reduced for the base area. The hybrid enucleoresection or resection according to Minervini's definition in the present study could be another reason for the higher renal function loss (35).

Cancer control is critical in the management of malignancies. In our report, no local recurrence was detected in all patients and only one metastasis was reported for one patient. This outcome suggested the efficacy of $\mathrm{PN}$ in cancer control. However, this conclusion should be cautiously interpreted. The positive surgical margin could be an important risk factor for recurrence (36). In our study, due to the relatively conservative resection strategy, all patients had a negative surgical margin. The efficacy of PN in cancer control still needs to be ascertained when simple enucleation is applied.

The choosing of procedures is a complicated issue in clinical practice. RN is still the most commonly used treatment for T2 renal tumors. Although PN could offer better renal function preservation, it requires a comprehensive preoperative assessment and an experienced team, and the surgeons and patients need to reach an agreement. Before surgery, imaging examinations including abdominal CT, renal angiogram, and MRI should be performed to evaluate the tumor complexity. As the central role in the procedure decision, the experienced surgeon would assess the feasibility and the potential risks of PN, and inform the patient of the merits and demerits of PN. The surgical infeasibility and potential risks prevented some patients from undergoing $\mathrm{PN}$, especially these patients with a better preoperative renal function. For these patients with a solitary kidney or poor preoperative renal function, PN might be more acceptable.

There was also a variety of strategies for PN. Open surgery, laparoscopic surgery, and robotic surgery were all widely used in urology. With the development of surgical instruments and the minimally invasive technology, laparoscopic surgeries were increasingly used in the field of surgery, especially in urology. Compared with open PN for T2 tumors, the laparoscopic PN could shorten the ischemia time and reduce the rate of intraoperative blood transfusion (22). RAPN also has some advantages over standard LPN. With higher magnification, the anatomical structures could be more clearly observed in RAPN. In the standard LPN, the operative angle was limited and the precise cutting could be difficult sometimes and this might explain the conversions to open surgery or RN during LPN. The robotic arm allows more flexible operative angles and a more stable incision and suturing, therefore the robotic surgery could be more favored by surgeons, especially in surgeries with high difficulty. The most PN for T2 tumors in 2017-2018 in our institution were conducted with RAPN also indicated such a trend. In our analysis, the operative time, blood loss, and hospital stay did not differ significantly between RAPN and LPN. The warm ischemia time seemed shorter in RAPN compared with LPN. However, because of the limited sample size, this comparison is not convincing enough, and better-designed studies with larger sample size are still warranted to ascertain whether robotic surgery can shorten the ischemia time.

This study has several limitations. First, the retrospective and single-institutional design might bring selective bias. For patients with high-complexity renal tumors, radical nephrectomy was more often recommended. Second, the limited follow-up of oncological outcomes and long-term renal functions were not enough for further assessment of these outcomes. Third, most LPNs were conducted in 2015-2016 and the RAPNs were more favored by surgeons latterly and the potential bias should be noted. Last, the 
small sample size also compromised the validity of the conclusions and we are unable to confirm the benefit of RAPN compared with LPN. Further studies with larger sample sizes were still warranted to better evaluate RAPN.

\section{Conclusions}

In conclusion, our single-institution experience suggested that RAPN might be a safe and effective procedure for renal tumors $\geq 7 \mathrm{~cm}$. It could provide great preservation of renal function and acceptable perioperative and oncological outcomes.

RAPN should be considered for patients with T2 tumors at an experienced medical institution.

\section{Acknowledgments}

Funding: This work is supported by the Natural Science Fund of Hubei Province (Grant Number: 2018CFB459).

\section{Footnote}

Reporting Checklist: The authors have completed the STROBE reporting checklist. Available at http://dx.doi. org/10.21037/tcr-20-2324

Data Sharing Statement: Available at http://dx.doi. org/10.21037/tcr-20-2324

Conflicts of Interest: All authors have completed the ICMJE uniform disclosure form (available at http://dx.doi. org/10.21037/tcr-20-2324). The authors have no conflicts of interest to declare.

Ethical Statement: The authors are accountable for all aspects of the work in ensuring that questions related to the accuracy or integrity of any part of the work are appropriately investigated and resolved. The study was conducted in accordance with the Declaration of Helsinki (as revised in 2013). The ethical approval of this study was exempted by the Ethics Committee of Tongji hospital because of the retrospective and observational nature, and the data were de-identified. Informed consent was also not required due to the above reasons.

Open Access Statement: This is an Open Access article distributed in accordance with the Creative Commons Attribution-NonCommercial-NoDerivs 4.0 International
License (CC BY-NC-ND 4.0), which permits the noncommercial replication and distribution of the article with the strict proviso that no changes or edits are made and the original work is properly cited (including links to both the formal publication through the relevant DOI and the license). See: https://creativecommons.org/licenses/by-nc-nd/4.0/.

\section{References}

1. Jemal A, Bray F, Center MM, et al. Global cancer statistics. CA Cancer J Clin 2011;61:69-90.

2. Ljungberg B, Campbell SC, Choi HY, et al. The epidemiology of renal cell carcinoma. Eur Urol 2011;60:615-21.

3. Campbell SC, Novick AC, Belldegrun A, et al. Guideline for management of the clinical T1 renal mass. J Urol 2009;182:1271-9.

4. Ljungberg B, Bensalah K, Canfield S, et al. EAU guidelines on renal cell carcinoma: 2014 update. Eur Urol 2015;67:913-24.

5. Van Poppel H, Da Pozzo L, Albrecht W, et al. A prospective, randomised EORTC intergroup phase 3 study comparing the oncologic outcome of elective nephronsparing surgery and radical nephrectomy for low-stage renal cell carcinoma. Eur Urol 2011;59:543-52.

6. Van Poppel H, Da Pozzo L, Albrecht W, et al. A prospective randomized EORTC intergroup phase 3 study comparing the complications of elective nephron-sparing surgery and radical nephrectomy for low-stage renal cell carcinoma. Eur Urol 2007;51:1606-15.

7. Zhang Y, Long G, Shang H, et al. Comparison of the oncological, perioperative and functional outcomes of partial nephrectomy versus radical nephrectomy for clinical T1b renal cell carcinoma: A systematic review and meta-analysis of retrospective studies. Asian J Urol 2019. doi: 10.1016/j.ajur.2019.11.004.

8. Badalato GM, Kates M, Wisnivesky JP, et al. Survival after partial and radical nephrectomy for the treatment of stage T1bN0M0 renal cell carcinoma (RCC) in the USA: a propensity scoring approach. BJU Int 2012;109:1457-62.

9. Meskawi M, Becker A, Bianchi M, et al. Partial and radical nephrectomy provide comparable long-term cancer control for T1b renal cell carcinoma. Int J Urol 2014;21:122-8.

10. Antonelli A, Ficarra V, Bertini R, et al. Elective partial nephrectomy is equivalent to radical nephrectomy in patients with clinical $\mathrm{T} 1$ renal cell carcinoma: results of a retrospective, comparative, multi-institutional study. BJU Int 2012;109:1013-8. 
11. Weight CJ, Larson BT, Fergany AF, et al. Nephrectomy induced chronic renal insufficiency is associated with increased risk of cardiovascular death and death from any cause in patients with localized cT1b renal masses. J Urol 2010;183:1317-23.

12. Jang HA, Kim JW, Byun SS, et al. Oncologic and Functional Outcomes after Partial Nephrectomy Versus Radical Nephrectomy in T1b Renal Cell Carcinoma: A Multicenter, Matched Case-Control Study in Korean Patients. Cancer Res Treat 2016;48:612-20.

13. Thompson RH, Siddiqui S, Lohse CM, et al. Partial versus radical nephrectomy for 4 to $7 \mathrm{~cm}$ renal cortical tumors. J Urol 2009; 182:2601-6.

14. Becker F, Roos FC, Janssen M, et al. Short-term functional and oncologic outcomes of nephron-sparing surgery for renal tumours $>/=7 \mathrm{~cm}$. Eur Urol 2011;59:931-7.

15. Breau RH, Crispen PL, Jimenez RE, et al. Outcome of stage T2 or greater renal cell cancer treated with partial nephrectomy. J Urol 2010;183:903-8.

16. Karellas ME, O'Brien MF, Jang TL, et al. Partial nephrectomy for selected renal cortical tumours of $>/=7$ cm. BJU Int 2010;106:1484-7.

17. Kopp RP, Liss MA, Mehrazin R, et al. Analysis of Renal Functional Outcomes After Radical or Partial Nephrectomy for Renal Masses $>/=7 \mathrm{~cm}$ Using the RENAL Score. Urology 2015;86:312-9.

18. Long CJ, Canter DJ, Kutikov A, et al. Partial nephrectomy for renal masses $>/=7 \mathrm{~cm}$ : technical, oncological and functional outcomes. BJU Int 2012;109:1450-6.

19. Song E, Ma X, An R, et al. Retroperitoneal Laparoscopic Partial Nephrectomy for Tumors Larger than 7 $\mathrm{cm}$ in Renal Cell Carcinoma: Initial Experience of Single-Institution. J Laparoendosc Adv Surg Tech A 2017;27:1127-31.

20. Bertolo R, Autorino R, Simone G, et al. Outcomes of Robot-assisted Partial Nephrectomy for Clinical T2 Renal Tumors: A Multicenter Analysis (ROSULA Collaborative Group). Eur Urol 2018;74:226-32.

21. Brandao LF, Zargar H, Autorino R, et al. Robotassisted partial nephrectomy for $>/=7 \mathrm{~cm}$ renal masses: a comparative outcome analysis. Urology 2014;84:602-8.

22. Malkoc E, Ramirez D, Kara O, et al. Robotic and open partial nephrectomy for localized renal tumors larger than $7 \mathrm{~cm}$ : a single-center experience. World J Urol 2017;35:781-7.

23. Gupta GN, Boris R, Chung P, et al. Robot-assisted laparoscopic partial nephrectomy for tumors greater than $4 \mathrm{~cm}$ and high nephrometry score: feasibility, renal functional, and oncological outcomes with minimum 1 year follow-up. Urol Oncol 2013;31:51-6.

24. Serni S, Vittori G, Frizzi J, et al. Simple enucleation for the treatment of highly complex renal tumors: Perioperative, functional and oncological results. Eur J Surg Oncol 2015;41:934-40.

25. von Elm E, Altman DG, Egger M, et al. The Strengthening the Reporting of Observational Studies in Epidemiology (STROBE) statement: guidelines for reporting observational studies. Ann Intern Med 2007;147:573-7.

26. Kutikov A, Uzzo RG. The R.E.N.A.L. nephrometry score: a comprehensive standardized system for quantitating renal tumor size, location and depth. J Urol 2009;182:844-53.

27. Levey AS, Coresh J, Balk E, et al. National Kidney Foundation practice guidelines for chronic kidney disease: evaluation, classification, and stratification. Ann Intern Med 2003;139:137-47.

28. Dindo D, Demartines N, Clavien PA. Classification of surgical complications: a new proposal with evaluation in a cohort of 6336 patients and results of a survey. Ann Surg 2004;240:205-13.

29. Mir MC, Derweesh I, Porpiglia F, et al. Partial Nephrectomy Versus Radical Nephrectomy for Clinical T1b and T2 Renal Tumors: A Systematic Review and Meta-analysis of Comparative Studies. Eur Urol 2017;71:606-17.

30. Deng W, Chen L, Wang Y, et al. Partial nephrectomy versus radical nephrectomy for large $(>/=7 \mathrm{~cm})$ renal tumors: A systematic review and meta-analysis. Urol Oncol 2019;37:263-72.

31. Wahafu W, Ma X, Li HZ, et al. Evolving renorrhaphy technique for retroperitoneal laparoscopic partial nephrectomy: single-surgeon series. Int J Urol 2014;21:865-73.

32. Go AS, Chertow GM, Fan D, et al. Chronic kidney disease and the risks of death, cardiovascular events, and hospitalization. N Engl J Med 2004;351:1296-305.

33. Antonelli A, Minervini A, Sandri M, et al. Below Safety Limits, Every Unit of Glomerular Filtration Rate Counts: Assessing the Relationship Between Renal Function and Cancer-specific Mortality in Renal Cell Carcinoma. Eur Urol 2018;74:661-7.

34. Minervini A, Campi R, Lane BR, et al. Impact of Resection Technique on Perioperative Outcomes and Surgical Margins after Partial Nephrectomy for Localized Renal Masses: A Prospective Multicenter Study. J Urol 
2020;203:496-504.

35. Minervini A, Carini M, Uzzo RG, et al. Standardized reporting of resection technique during nephron-sparing surgery: the surface-intermediate-base margin score. Eur Urol 2014;66:803-5.

Cite this article as: Long G, Liu M, Zhang Y, Sun G, Ouyang W, Yang J, Wang Z, Liu Z, Guan W, Hu Z, Wang S, Li H. Robot-assisted laparoscopic partial nephrectomy is a safe and effective option for clinical T2 renal cell carcinoma: a caseseries from single-institution. Transl Cancer Res 2020;9(11): 7140-7148. doi: 10.21037/tcr-20-2324
36. Khalifeh A, Kaouk JH, Bhayani S, et al. Positive Surgical Margins in Robot-Assisted Partial Nephrectomy: A MultiInstitutional Analysis of Oncologic Outcomes (Leave No Tumor Behind). J Urol 2013;190:1674-9. 